\title{
Análise do teste de dose de entrada na pele realizados em serviços de radiodiagnóstico médico do estado de Minas Gerais
} Analysis of the entrance surface dose tests performed in diagnostic radiology services of Minas Gerais state, in Brazil.

\author{
Fábio Sabará Dias ${ }^{1}$, Paulo Márcio Campos Oliveira² ${ }^{2}$ Leandro de Abreu Vieira ${ }^{3}$, Marco \\ Aurélio de Sousa Lacerda1. \\ ${ }^{1}$ Centro de Desenvolvimento da Tecnologia Nuclear, Belo Horizonte, Brasil \\ ${ }^{2}$ Anatomia e Imagem, Universidade Federal de Minas Gerais, Belo Horizonte, Brasil \\ ${ }^{3}$ Secretária da Saúde do Estado de Minas Gerais, Belo Horizonte, Brasil
}

\begin{abstract}
Resumo
Neste trabalho foi realizado o estudo retrospectivo acerca da análise do teste de dose de entrada na pele (DEP) realizado por profissionais credenciados pela VISA/MG em clínicas de radiodiagnóstico médico. Foi avaliada a dose recebida pelos pacientes em um período retrospectivo de 5 anos, nos exames radiográficos mais frequentemente realizados em instalações de Minas Gerais. Esses valores de DEP foram comparados com os níveis de referência em radiodiagnóstico (NRDs) recomendados pela Portaria 453/98. Uma comparação com normas internacionais também foi realizada, com intuito de verificar se os NRDs recomendados por órgãos internacionais provocaria um alto índice de não conformidade no teste. Do total de testes estudados, $75 \%$ estão em conformidade com os NRDs estabelecidos pela Portaria 453/98. Para o exame de coluna lombar e crânio, os valores médios de DEP foram cerca de duas vezes menores que os NRDs recomendados. Para o exame de tórax, foi possível verificar que as médias das doses de entrada na pele são bem similares em todo o período estudado, tendo como média geral um valor de $0,25 \mathrm{mGy}$. Ao comparar o exame de tórax com os NRDs propostos por normas internacionais, obteve-se uma queda de $14 \%$ na conformidade. $O$ alto índice de conformidade sugere que as técnicas radiográficas selecionadas foram adequadas para obtenção de doses menores que os níveis de referência estabelecidos.
\end{abstract}

Palavras-chave: proteção radiológica; controle de Qualidade; radiodiagnóstico Médico.

\begin{abstract}
In this work, we carried out a retrospective study on the analysis of the entrance surface dose (ESD) test performed by professionals accredited by the VISA / MG in medical diagnostic radiology clinics. We evaluated the ESD received by patients in a retrospective period of 5 years, in the most frequent radiographic examinations performed at facilities in Minas Gerais state, Brazil. ESD values were compared with the Diagnostic Reference Levels (DRLs) recommended by the Brazilian current legislation. A comparison with international standards was also carried out in order to verify if the DRLs recommended by these international bodies would cause a high rate of non-conformity in the test. From the total number of DRLs values reported, $75 \%$ comply with the DRLs established by the Brazilian norm. For lumbar spine and head examinations, mean values of DRLs were twice lower than the recommended DRLs. For chest examinations, mean ESD values were similar for all the period studied. The overall average, in this case, was $0.25 \mathrm{mGy}$. For the chest exam, a reduction of $14 \%$ in compliance is verified when compared with the DRLs established by international standards. The high compliance rates suggest that radiographic techniques selected were suitable for obtaining doses lower than the DRLs established by Brazilian legislation.
\end{abstract}

Keywords: radiation protection; quality control; medical diagnostic radiology.

\section{Introdução}

De acordo com uma publicação da United Nations Scientific Committee on the Effects of Atomic Radiation (UNSCEAR), são realizados aproximadamente 3,6 bilhões de exames diagnósticos, anualmente, em todo o mundo, resultando em uma dose efetiva de 0,6 mSv/ano, aproximadamente ${ }^{1}$.

A Comissão Internacional de Proteção Radiológica (ICRP) não recomenda a aplicação de limites de dose para os pacientes submetidos a exames de radiodiagnóstico, pois, uma vez que esses exames sejam clinicamente justificados, os benefícios para os pacientes serão sensivelmente superiores aos riscos radioinduzidos ${ }^{2,3}$. No entanto, como forma de otimizar o uso das radiações ionizantes para essa finalidade, foi adotado pela ICRP, nas publicações 60 e $73^{4,5}$ o conceito de doses de referência, em exames radiológicos frequentemente realizados. $\mathrm{Na}$ publicação 73 da ICRP foi introduzido o termo Nível de Referência em Radiodiagnóstico (NRD) ${ }^{5}$. Nessa publicação, a ICRP recomenda que os valores de NRDs devem ser selecionados por organismos médicos profissionais e serem específicos para um país ou região. Além disso, os NRDs devem ser revistos em intervalos de tempo que representem um 
compromisso entre a estabilidade necessária e as mudanças a longo prazo nas distribuições das doses observadas. Os níveis de referência em radiodiagnóstico (NRDs) são níveis orientativos. Caso se comprove que eles estão sendo sistematicamente excedidos em procedimentos radiográficos realizados na instituição, deve-se efetuar uma reavaliação dos procedimentos e / ou do equipamento $\mathrm{e}$, se necessário, adotar medidas corretivas $^{2,3}$

Os valores NRDs são baseados em pesquisas para cada modalidade de exames em radiodiagnóstico e medidas são realizadas em uma amostra apropriada de pacientes. Vale ressaltar que não é recomendado o uso de "phantoms", que na maioria dos casos não é suficiente, já que os efeitos do desempenho do operador e alguns outros fatores, não são levados em conta ${ }^{2}$

No Brasil, a Portaria SVS-MS 453/986 ${ }^{6}$, determina as diretrizes básicas de proteção radiológica em radiodiagnóstico médico e odontológico. Tal legislação estabelece que todos os serviços de radiodiagnóstico médico devem implementar um Programa de Controle de Qualidade (PGQ) e que o mesmo deve ser monitorado e/ou fiscalizado através de testes periódicos que avaliam o desempenho do equipamento e a estrutura do ambiente ao qual ele se insere ${ }^{6}$.

A Portaria SVS-MS 453/98 ainda propõe níveis de referência em radiodiagnóstico para uma série de exames e posicionamento, sendo que estes valores de NRDs referem-se a combinações tela-filme regulares (velocidade relativa 200). No entanto, esses NRDs foram baseados em publicações internacionais e não em levantamentos dosimétricos realizados em instalações radiológicas brasileiras. Esses valores de NRDs ainda se referem apenas para sistemas tela-filme sendo que nos últimos 20 anos a tecnologia avançou e valores de referência atualizados devem ser adotados.

Como complemento à Portaria SVS-MS 453/98, foi publicado em 2005, pela editora ANVISA, um guia intitulado "Radiodiagnóstico Médico: Desempenho de Equipamentos e Segurança"7, com o objetivo de padronizar os procedimentos para a realização dos testes de controle de qualidade nos estabelecimentos e equipamentos de raios $X$. Tal guia fornece a metodologia que pode ser aplicada para a realização dos testes de constância exigidos pela norma vigente ${ }^{7}$.

Um dos testes exigidos é o de Dose de Entrada na Pele (DEP). Assim, a DEP deve ser estimada ou avaliada para os exames mais frequentemente realizados na instalação e comparados aos NRDs propostos pela Portaria SVS-MS 453/98.

Em Minas Gerais, o órgão de Vigilância Sanitária estadual (VISA-MG) é responsável pelo cadastramento dos profissionais responsáveis pela realização dos testes exigidos por norma ${ }^{8}$. Esses profissionais são obrigados a submeter seus relatórios para a VISA-MG.

O objetivo desse trabalho é avaliar, a partir dos relatórios submetidos pelos profissionais credenciados pela VISA-MG, a DEP nos exames mais frequentemente realizados em instalações de Minas Gerais. Assim, esses valores de Dose na Entrada da Pele foram comparados com os NRDs propostos pela Portaria SVS-MS $453 / 98^{6}$ e normas internacionais.

\section{Materiais e Métodos}

Os relatórios de testes de constância disponibilizados pela VISA/MG em um período retrospectivo de 5 anos, 2011 a 2015, foram avaliados. Os dados apresentados possuem informações de equipamentos distribuídos por todo o estado de Minas Gerais, nesse período.

É importante salientar que segundo o guia da ANVISA $^{7}$ é recomendada a realização do teste para 4 exames: coluna lombar (AP, LAT e JLS), tórax (PA e LA), abdômen (AP) e crânio (AP e Lateral). Entretanto, não foi possível fazer a análise para todos os exames e/ou incidências já que 6 de 8 dos credenciados analisados, não seguem por completo a recomendação da publicação da ANVISA.

Os valores de DEP reportados nos relatórios foram comparados com os níveis de referência propostos pela Portaria SVS-MS 453/98 e Comunidade Europeia $(\mathrm{CE})^{9}$. A Tabela 1 mostra os valores recomendados pelas normas já citadas dos níveis de referência, para adulto padrão (massa de 60 a 70 kg e altura de 160 a $175 \mathrm{~cm}$ ) e para combinações telafilme regulares (velocidade relativa 200) ${ }^{6,7}$.

Tabela 1. Níveis de referência em radiodiagnóstico por radiografia para paciente adulto típico.

\begin{tabular}{ccc}
\hline Exame & Incidência & DEP (mGy) \\
\hline Coluna Lombar & AP & 10 \\
Tórax & PA & $0,4 / 0,3^{*}$ \\
Crânio & AP & 5 \\
\hline Nota: * corresponde ao nível de referência recomendado pela CE. \\
AP é a incidência Anteroposterior & \\
PA é a incidência Póstero-anterior &
\end{tabular}

\section{Resultados e Discussões}

Foram avaliados 385 relatórios de testes de constância. A Figura 1 mostra o número de relatórios que tiveram todas as incidências analisadas neste trabalho com resultados satisfatórios (DEP inferior ao NRD proposto pela Portaria SVS-MS 453/98), os que tiveram ao menos uma incidência com resultado insatisfatório e os relatórios em que o teste não foi realizado.

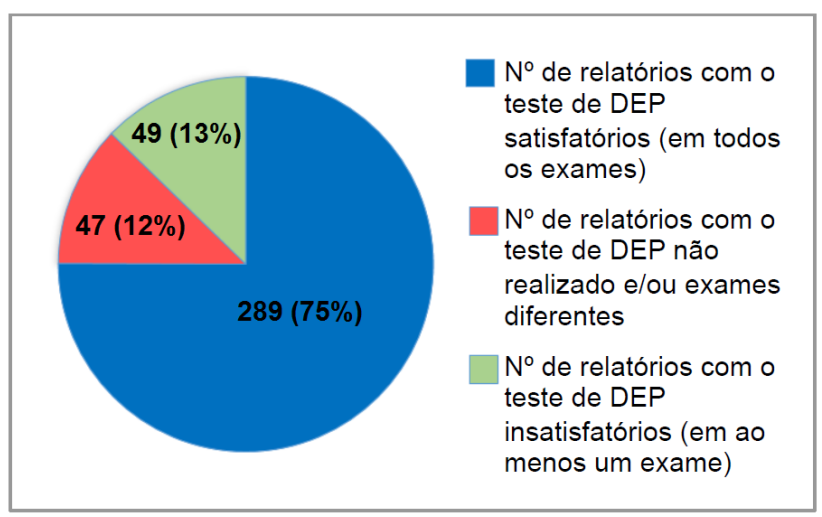

Figura 1. Avaliação do teste de dose de entrada na pele no estado de Minas de Gerais, de 2011 a 2015. 
Através da Figura 1 foi possível observar que cerca de $75 \%$ dos testes encontra-se em conformidade, $\mathrm{o}$ que indica que as técnicas selecionadas pelos profissionais de radiologia estão apropriadas e os aparelhos estão respondendo adequadamente. Tem-se um número baixo de testes não conformes, aproximadamente $13 \%$, sendo que não houve relatório com os três exames, com suas respectivas incidências, com desempenhos insatisfatórios. O restante, $12 \%$, corresponde aos relatórios em que o teste não foi reportado ou que possuíam exames diferentes. Estão incluídos neste último caso, 32 relatórios, em que foram reportados os valores de DEP para o exame de abdome (AP). Entretanto, deve-se ressaltar que o ideal seria a realização dos testes para todos os exames recomendados na publicação da ANVISA.
É importante salientar que para cada tipo de exame estudado, tórax (PA), coluna lombar (AP) e crânio (AP), obteve-se uma conformidade superior a $90 \%$ dos testes analisados.

Os próximos dados são referentes à distribuição dos valores de dose encontrados nos relatórios para cada tipo de exame separadamente, de um modo geral e para cada ano estudado. Para as distribuições que tem como variável o ano, não foi possível obter informações para o ano de 2013, pois o mesmo só apresenta um valor de DEP encontrado para cada exame.

A Figura 2 mostra a distribuição dos valores de dose de entrada na pele para o exame de coluna lombar (AP). A Tabela 2 mostra os valores de mínimo, média e máxima, encontrados na distribuição da DEP para o exame de coluna lombar.

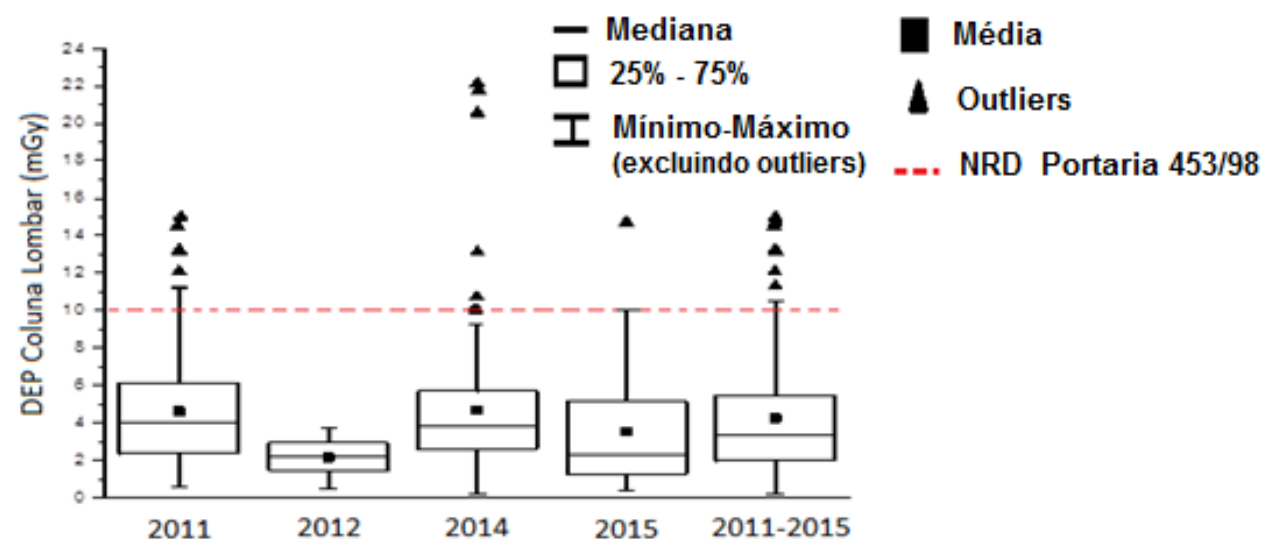

Figura 2. Distribuição dos valores de DEP para o exame de coluna lombar (AP) para cada ano

Tabela 2. Valores mínimos, médios e máximos da DEP ara o exame de coluna lombar.

\begin{tabular}{lccccc}
\hline \multirow{5}{*}{$\begin{array}{c}\text { DEP } \\
(\mathrm{mGy})\end{array}$} & 2011 & 2012 & 2013 & 2014 & $\begin{array}{c}2011- \\
2015\end{array}$ \\
\cline { 2 - 6 } & 0,53 & 0,50 & 0,18 & 0,41 & 0,18 \\
\hline Mínimo & 4,62 & 2,13 & 4,69 & 3,53 & 4,27 \\
Média & 4,62 & 22,13 & 14,71 & 22,13 \\
\hline
\end{tabular}

Através dos dados mostrados na Figura 2 e na Tabela 2 é verificado que durante o período estudado, os valores médios de DEP para o exame de coluna lombar foram cerca de duas vezes menores que o NRD recomendado pela Portaria SVS-MS 453/98.

Os valores dos 3 Qs estão abaixo do estabelecido na norma vigente, com o valor de 5,74 mGy para o período total estudado. O maior valor encontrado foi no ano de 2011, 6,10 mGy, que é 39\% inferior ao NRD. Já o intervalo interquartil (IQR) do período estudado é de 3,77 mGy, tendo os anos de 2012 e $2014 \mathrm{com}$ valores mais distantes, respectivamente 1,40 e 2,93 mGy, indicando que estes anos tiveram menores variações nos valores de DEP.

Observa-se que em todos os anos e na avaliação do período geral, a distribuição é positivamente assimétrica, com maior grau de assimetria no ano de 2015, onde a mediana, 2,32 mGy, está bem abaixo da média 3,53mGy.
A mediana do período estudado teve um valor de 3,38 mGy. Este resultado está $66,2 \%$ abaixo do nível de referência proposto na norma vigente. $\mathrm{O}$ ano de 2011, foi o que teve maior mediana, 4,04 mGy, valor este que está $59,6 \%$ abaixo do NRD estabelecido.

Para o ano de 2012, foi observada a menor média e dispersão dos valores de DEP. Este resultado pode ser justificado pelo fato de haver um menor número de dados coletados para este ano, ressaltando que é possível manter as doses bem abaixo dos NRDs estabelecidos, desde que não afete a qualidade da imagem.

Os valores de mediana encontrados abaixo $25 \%$ da dose de referência analisados para cada exame, devem ser avaliados separadamente, analisando prioritariamente a qualidade da imagem, ao invés do nível de dose de entrada na pele para o paciente típico, sendo útil no processo de otimização do serviço ${ }^{2}$.

Contabilizou-se um número de 10 outliers para o período estudado, como mostra a Figura 2. Em alguns casos, esses outliers foram mais de duas vezes superior ao NRD proposto, indicando que pode haver falhas nos aparelhos e/ou utilização de técnicas inadequadas por parte dos técnicos ou tecnólogos de radiologia. 
A Figura 3 mostra a distribuição da DEP para o exame de tórax (PA), que possui um valor de NRD bem abaixo dos demais, 0,4 mGy. A Tabela 3 mostra os valores de mínimo, média e máxima encontrada na distribuição da DEP (PA) para o exame de tórax.

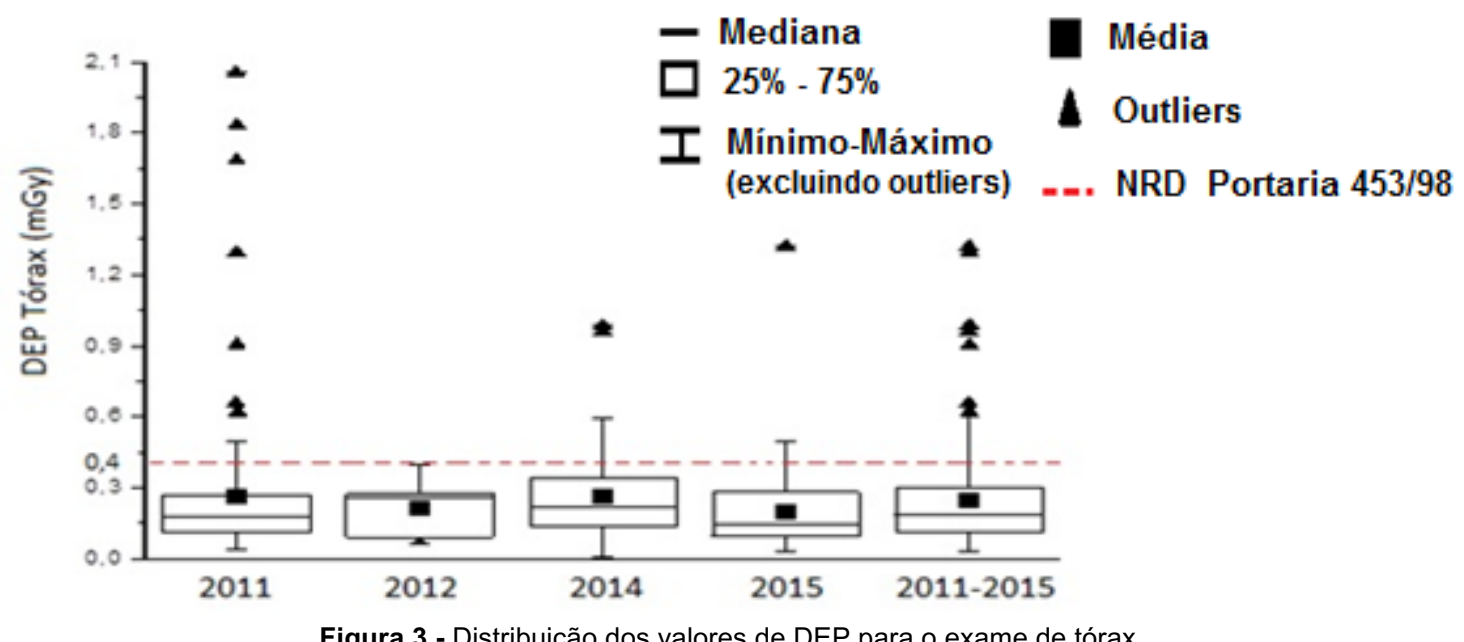

Tabela 3- Valores mínimos, médios e máximos da DEP para o exame de tórax.

\begin{tabular}{cccccc}
\hline \multirow{5}{*}{$\begin{array}{c}\text { DEP } \\
(\mathrm{mG} y)\end{array}$} & 2011 & 2012 & 2013 & 2014 & $\begin{array}{c}2011- \\
2015\end{array}$ \\
\cline { 2 - 6 } & 0,01 & 0,06 & 0,01 & 0,03 & 0,01 \\
Mínimo & 0,27 & 0,21 & 0,26 & 0,2 & 0,25 \\
Média & 0,26 & 0,98 & 1,32 & 2,17 \\
Máximo & 2,17 & 0,40 & 0,903 \\
\hline
\end{tabular}

Através dos dados mostrados na Figura 3 e na Tabela 3, foi possível verificar que as médias das doses de entrada na pele são bem similares, tendo como média geral um valor de 0,25 mGy. Isto indica que as técnicas radiográficas selecionadas foram adequadas para obtenção de doses menores que os níveis de referência estabelecidos. Entretanto, não se pode afirmar que com essas técnicas radiográficas é possível obter imagens de boa qualidade diagnóstica.

Os 3Qs observados foram bem próximos e os valores bem abaixo dos NRDs estabelecidos. O valor de $3 \mathrm{Q}$ para todo o período, 0,27 $\mathrm{mGy}$, é 32,5\% menor que os NRD estabelecido. Apenas no ano de 2014, o 3Q foi superior, 0,34 mGy. No entanto, esse valor é $15 \%$ menor que o NRD vigente.

Os IQRs também tiveram valores próximos, variando de 0,16 a 0,20 mGy, indicando uma baixa dispersão dos valores encontrados.

Apenas no ano de 2012, todos os dados estiveram dentro dos NRDs estabelecidos pela Portaria SVSMS 453/98, com a ressalva de que foi o ano com menos dados analisados.

Para o período total analisado é mostrado uma distribuição simétrica, com uma pequena diferença entre a mediana e média, 0,06 mGy. Já no ano de 2012 é observada uma distribuição negativamente assimétrica, com sua mediana acima do valor médio e mais próximo do $3 \mathrm{Q}$. Nos demais anos, é observado uma distribuição positivamente assimétrica, com o ano de 2015 sendo mais acentuado.

Foi contabilizado um número alto de outliers superiores, que assim como alguns valores acima do $3 Q$, estão ultrapassando os NRDs. Em alguns casos, esses outliers foram cerca de cinco vezes superior ao NRD proposto. Estes valores discrepantes podem indicar não só a utilização de técnicas inadequadas, mas também a presença de falhas nos aparelhos.

Ao analisar os resultados utilizando o valor de referência da Comunidade Europeia que é de $\quad 0,3$ mGy para o exame de tórax ${ }^{7}$, dos 327 resultados reportados, 243 (74\%) serviços de radiodiagnóstico teriam resultados satisfatórios. É apurada uma queda de $17 \%$ de conformidade se comparada com as recomendações da Portaria SVS-MS 453/98. Ainda assim, é verificado um valor alto de testes de DEP satisfatórios, indicando que é plausível uma diminuição maior dos níveis de referência estabelecidos, já que mais de $70 \%$ dos serviços de radiodiagnostico já conseguem implementar o mesmo.

A Figura 4 mostra a distribuição dos valores de dose de entrada na pele para o exame de crânio (AP). A Tabela 3 mostra os valores de mínimo, média e máximo encontrados na distribuição da DEP para o exame de coluna cujo NRD é 5 mGy. 


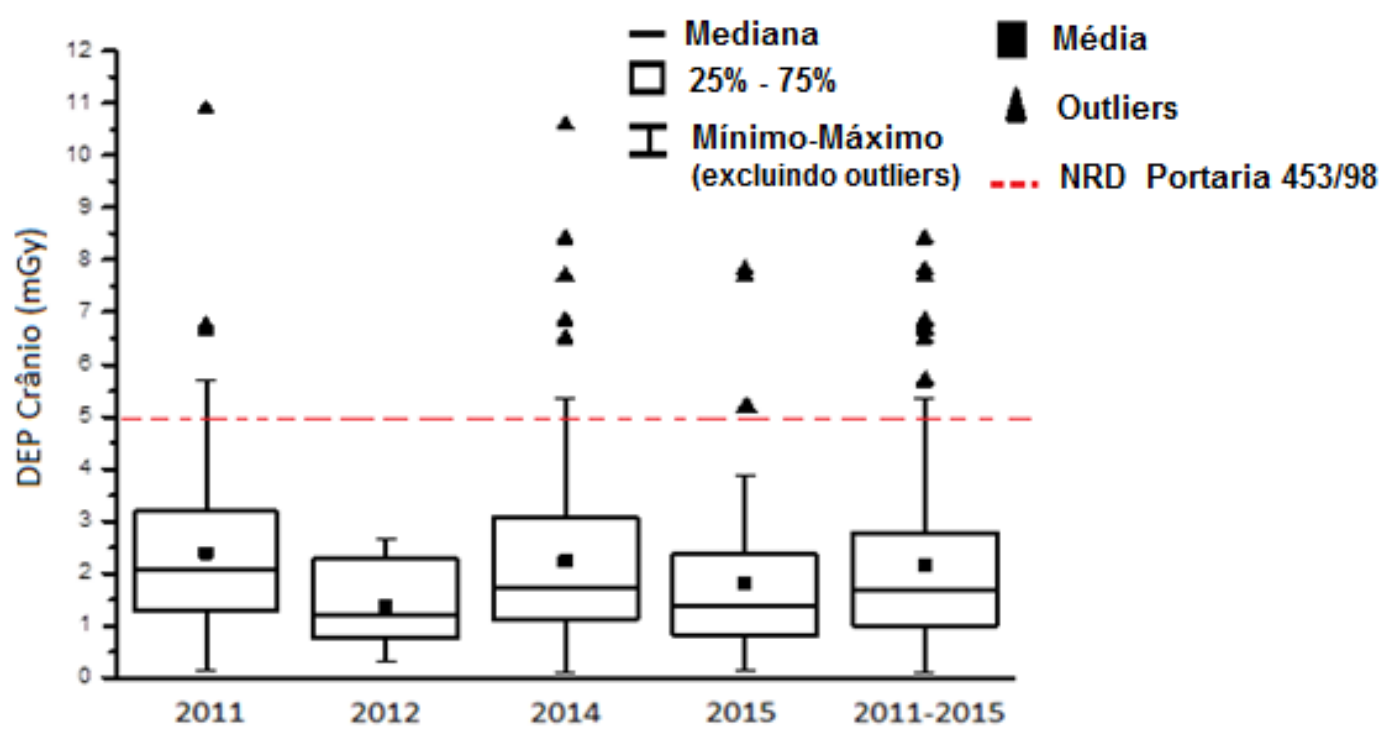

Figura 4 - Distribuição dos valores de DEP para o exame de crânio (AP).

Tabela 4- Valores mínimos, médios e máximos da DEP para o exame de crânio (AP).

\begin{tabular}{cccccc}
\hline \multirow{5}{*}{$\begin{array}{c}\text { DEP } \\
\text { (mGy) }\end{array}$} & 2011 & 2012 & 2013 & 2014 & $\begin{array}{c}2011- \\
2015\end{array}$ \\
\cline { 2 - 6 } & 0,13 & 0,3 & 0,11 & 0,16 & 0,11 \\
Mínimo & $0,1,39$ & 2,24 & 1,81 & 2,21 \\
Média & 2,39 & 1,39 & 10,59 & 7,82 & 10,89 \\
Máximo & 10,89 & 2,67 & 10
\end{tabular}

De acordo com a Figura 4 e a Tabela 4 foi possível verificar que os valores médios de DEP para o exame de crânio foram, aproximadamente, 2 vezes menores que o NRD recomendado na Portaria SVS-MS $453 / 98$.

Os 3Qs também tiveram valores abaixo do recomendado pela norma vigente, respectivamente, $3,16,1,99,3,06,2,36 \mathrm{mGy}$ para cada ano. Já o valor encontrado no período de 2011 a 2015 , está $37,3 \%$ abaixo do NRD estabelecido.

No período avaliado, entre os anos de 2011 e 2015, o IQR foi de 1,90 mGy. Já os anos de 2012 e 2015 tiveram valores abaixo, respectivamente 1,12 e 1,56 $\mathrm{mGy}$, indicando que estes foram os anos com menores discrepâncias entre os valores de DEP.

Apenas os anos de 2012 e 2015 apresentaram toda a distribuição dos valores, excluindo os outliers, abaixo dos NRDs estabelecido pela Portaria SVS-MS 453/98. Logo, estes foram os anos com uma melhor avaliação de DEP para o exame de crânio.

No período geral estudado a distribuição é positivamente assimétrica com uma diferença de 0,44 mGy entre a mediana e a média. Já em cada ano avaliado, a distribuição também é positivamente assimétrica, sendo o ano de 2014 mais acentuado.

Foi contabilizado um número alto outliers superiores, sendo que em sua maioria, possuem o dobro da dose de referência. Estes valores discrepantes podem indicar técnicas radiológicas inadequadas e/ou não conformidade do aparelho.

Cabe aqui ressaltar importância que os serviços de radiodiagnóstico, assim como os credenciados e a própria VISA devem ter ao avaliar as doses nos exames, das mais altas até as mais baixas, associando-as à qualidade das imagens para fins de se implementar um programa de otimização.

Através da atualização recente da Resolução SES $n^{\circ} 5177$ de $2016^{8}$, é esperado uma evolução na conduta dos profissionais credenciados pela VISA/MG, uma vez que é possível a utilização de metodologias nacionais e internacionais para a realização e análise dos testes de constância. Com estas alterações, almeja-se uma melhora na qualidade dos relatórios e das análises realizadas pelos credenciados, assim como uma avaliação mais criteriosa dos equipamentos.

\section{Conclusões}

Os dados coletados e disponibilizados pela Vigilância Sanitária de Minas Gerais atenderam aos propósitos deste trabalho de maneira satisfatória.

Apenas $25 \%$ dos credenciados realizam as avaliações de DEP para os exames e incidências referidas na publicação da ANVISA (2005) e na Portaria SVS-MS 453/98.

Os exames mais reportados para esta avaliação são os de coluna lombar (AP), tórax (PA) e crânio (AP), que obtiveram uma conformidade superior a $90 \%$. Esta alta conformidade mostra que as técnicas radiográficas selecionadas foram adequadas para obtenção de doses menores que os níveis de referência estabelecidos.

Quando comparado com o protocolo europeu, foi verificado que aproximadamente $74 \%$ dos exames de tórax estariam em conformidade. Logo, é possível avaliar uma possível mudança nos níveis de referência estabelecidos na Portaria SVS-MS 453/98, se adequando a atualizações realizadas em diversos outros países.

Os valores encontrados abaixo das medianas analisadas para cada exame, devem ser melhor estudados, tendo a qualidade da imagem como fator chave na sua avaliação. Através desta análise adicional, é que será possível verificar se é possível manter doses tão baixas sem comprometer a qualidade do exame. 


\section{Agradecimentos}

Fábio Sabará Dias é grato ao CAPES, pela bolsa de mestrado e também a VISA-MG pela parceria e envio do material para este trabalho. Marco Aurélio de Sousa Lacerda é grato ao CNPq (MCTI/CNPq/Universal Proc. 449199/2014-2) e FAPEMIG (PPM-00208-15), pelo apoio financeiro.

\section{Referências}

1. UNSCEAR. Sources and effects of ionization radiation. v. 1 - Sources, Report to General Assembly, with Scientific Annexes, New York, 2008.

2. ICRP - International Commission on Radiological Protection. The 2017 Diagnostic reference levels in medical imaging. ICRP Publication 135. Ann. ICRP 46(1). ICRP 2017.

3. International Atomic Energy Agency. Radiation Protection and Safety of Radiation Sources: International Basic Safety Standards (Interim Edition), General Safety Requirements Part 3 No. GSR Part 3 (Interim). Vienna: IAEA; (2014).

4. ICRP - International Commission on Radiological Protection. 1990 Recommendations of the International Commission on Radiological Protection: adopted by the Commission in November 1990. Oxford, 1991. 201p. (ICRP 60)

5. ICRP - International Commission on Radiological Protection. Radiological protection and safety in medicine. STOCKHOLM: ICRP, 1996. 50 p. (ICRP-73).

6. BRASIL. MINISTÉRIO DA SAÚDE - ANVISA. Portaria 453 do Ministério da Saúde: Diretrizes de Proteção Radiológica em Radiodiagnóstico Médico e Odontológico. Diário Oficial da União de 02 de junho de 1998.

7. BRASIL. MINISTÉRIO DA SAÚDE - ANVISA. Radiodiagnóstico Médico: Desempenho de Equipamentos e Segurança / Ministério da Saúde. Agência Nacional de Vigilância Sanitária. Brasília, 2005.

8. MINAS GERAIS. SECRETARIA DE SAÚDE DO ESTADO DE MINAS GERAIS. Resolução SES $n^{\circ} 5177,2016$. Dispõe sobre o cadastramento de consultor externo no Estado de Minas Gerais, de empresa e/ou profissional para o exercício dos serviços de avaliação de equipamentos de raios $X$ e de ambientes na área de proteção radiológica em radiologia médica e odontológica. Disponível em: http://www.saude.mg.gov.br/images/documento s/resolu\%C3\%A7\%C3\%A3o 5177.pdf. Acesso em: 01/12/2017.

9. EUR. European Commission. European guidelines on quality criteria for diagnostic radiographic images. Office for Official Publications of the European Communities, Luxembourg, EUR16260EN, 1996a.
Fábio Sabará Dias

Av. Presidente Antônio Carlos, 6.627. Campus da UFMG - Pampulha, CEP 31270-901, Belo Horizonte - Minas Gerais, Brasil. E-mail: diasfs@cdtn.br.

\section{Contato:}

